\title{
Avaliação dos Movimentos Gerais de Prechtl (GMA) na deteç̧ão precoce de risco ao desenvolvimento
}

\author{
Prechtl's General Movements Assessment (GMA) in early detection for child development risk \\ Evaluación de los Movimientos Generales de Prechtl (GMA) en la detección precoz del riesgo \\ de desarrollo
}

\author{
Sabrina Felin Nunes ${ }^{1}$, Eloá Maria dos Santos Chiquetti², Anaelena Bragança de Moraes ${ }^{3}$, Ana Paula \\ Ramos de Souza ${ }^{4}$
}

RESUMO I Verificar a relação entre a avaliação dos movimentos gerais (General Movements Assessment - GMA) com as variáveis obstétricas (aleitamento materno, intercorrência na gestação, medicação na gestação, álcool na gestação, fumo na gestação, intercorrência ao nascer, necessidade de internação em UTI neonatal e necessidade de ventilação mecânica), a presença de risco psíquico e o desfecho no desenvolvimento da linguagem, cognitivo e motor aos 18 e 24 meses. A amostra foi composta por 42 bebês, que foram filmados até a faixa etária de quatro meses, em movimentação espontânea por 15 minutos. Os movimentos gerais foram avaliados por vídeos usando a avaliação qualitativa de Prechtl e classificados como normais ou anormais dependendo da presença de fluência, complexidade e variabilidade. Os dados foram analisados estatisticamente em sua relação com variáveis obstétricas e com a presença de risco psíquico, avaliada por meio dos Sinais PREAUT, dos indicadores clínicos de risco ao desenvolvimento e do M-CHAT. Verificou-se que não houve associação entre o método GMA e as variáveis analisadas. Acredita-se que, pelo fato de a amostra ter sido composta, em sua maioria, por bebês nascidos a termo ou prematuros tardios sem intercorrências e de a avaliação ter sido de forma transversal, em um único momento, não foi possível analisar se os movimentos avaliados como anormais foram ou não transitórios. A alteração dos movimentos por meio do método Prechtı não apresentou associação com as variáveis analisadas na amostra de bebês prematuros tardios e nascidos a termo. Descritores | Desenvolvimento Infantil; Medição de Risco; Fatores de Risco; Transtornos dos Movimentos; Avaliação da Deficiência.
ABSTRACT I To verify the relationship between the general movements assessment (GMA) and obstetric variables (maternal breastfeeding, pregnancy complications, gestational medication, alcohol consumption during gestation, smoking during gestation, intercurrence at birth, need for neonatal ICU admission, and need for mechanical ventilation), the presence of psychological risk and the outcome in language, cognitive, and motor development at 18 and 24 months. In total, 42 infants composed the sample and were filmed until the age of four months, in spontaneous movement for 15 minutes. The general movements were evaluated by videos using the qualitative evaluation of Prechtl and they were classified as normal or abnormal depending on the presence of fluency, complexity, and variability. The data were statistically analyzed regarding obstetric variables and the presence of psychological risk evaluated by the PREAUT signs, clinical indicators of developmental risk and M-CHAT. It was verified that there was no association between the GMA method and the analyzed variables. It is believed that because the sample was mostly composed of full-term infants or late premature infants without intercurrence, and the evaluation was transverse in a single moment, it was not possible to analyze whether the movements evaluated as abnormalities were or were not transient. The change of movements by the Prechtl method was not associated with the variables analyzed in the sample of late and term premature infants.

Keywords | Child Development; Risk Assessment; Risk Factors; Movement Disorders; Disability Evaluation.

Universidade Federal de Santa Maria (UFSM) - Santa Maria (RS), Brasil. E-mail: sah_felin@hotmail.com. Orcid: 0000-0001-5976-9760 2Universidade Federal do Pampa (Unipampa) - Uruguaiana (RS), Brasil. E-mail: eloachiquetti@unipampa.edu.br. Orcid: 0000-0003-1550-6373 3 Universidade Federal de Santa Maria (UFSM) - Santa Maria (RS), Brasil. E-mail: moraesanaelena@gmail.com. Orcid: 0000-0002-6578-0613 ${ }^{4}$ Universidade Federal de Santa Maria (UFSM) - Santa Maria (RS), Brasil. E-mail: ana.souza@ufsm.br. Orcid: 0000-0003-4733-0632 
RESUMEN I Este estudio tiene como objetivo verificar la relación entre la evaluación de los movimientos generales (GMA) con las variables obstétricas (lactancia materna, complicaciones del embarazo, medicación durante el embarazo, alcohol en el embarazo, tabaquismo durante el embarazo, complicaciones al nacer, necesidad de ingreso en la UCI neonatal y necesidad de mecánica ventilatoria), la presencia de riesgo psicológico y el resultado en el desarrollo del lenguaje, cognitivo y motor en bebés entre los 18 y 24 meses. La muestra constó de 42 bebés y se filmaron sus movimientos espontáneos por 15 minutos hasta los cuatro meses de edad. Los movimientos generales se determinaron por la técnica de evaluación cualitativa de Prechtl, los cuales se clasificaron como normales o anormales según la presencia de fluidez, complejidad y variabilidad.
Los datos se analizaron estadísticamente con relación a las variables obstétricas y la presencia de riesgo psíquico, evaluadas por los Señales PREAUT, Ios indicadores clínicos de riesgo al desarrollo y el M-CHAT. Se encontró que no hubo asociación entre el método GMA y las variables analizadas. Debido a que la muestra estuvo compuesta mayoritariamente por bebés nacidos a término o prematuros tardíos sin complicaciones y que la evaluación fue transversal, en un solo momento, no fue posible analizar si los movimientos evaluados como anormales fueron transitorios o no. Los cambios en los movimientos mediante el método de Prechtl no se asociaron con las variables analizadas en la muestra de prematuros tardíos y nacidos a término. Palabras clave | Desarrollo Infantil; Medición de Riesgo; Factores de Riesgo; Trastornos del Movimiento; Evaluación de la Discapacidad.

\section{INTRODUÇÃO}

Depois do nascimento, os quatro primeiros anos de uma criança são vistos como críticos para o estabelecimento de uma base sólida para o seu desenvolvimento posterior. A maioria das crianças apresenta um desenvolvimento normal, geralmente precisando somente de consultas de rotina com o pediatra. Entretanto, o desenvolvimento inicial de um subgrupo de crianças é pontuado por atrasos, que, se negligenciados, podem dar origem a um curso posterior de dificuldades ou distúrbios do desenvolvimento, que demandam atenção especializada de profissionais de referência ${ }^{1}$.

Vários fatores podem influenciar o desenvolvimento do bebê antes, durante e após seu nascimento. Dentre os fatores de risco biológico, o destaque é para o nascimento prematuro, uma vez que geralmente vem acompanhado de baixo peso ao nascimento, tempo de internação na unidade de terapia intensiva neonatal (UTIN) e uso da ventilação mecânica (VM). Entre os fatores ambientais que podem influenciar negativamente no desenvolvimento futuro da criança estão a baixa escolaridade materna e renda familiar, além de condições gestacionais e emocionais, como humor materno alterado, interação insuficiente da mãe com o bebê e baixa oferta de estímulos e brinquedos ao bebê $\hat{}^{1}$. Esses fatores enfatizam a importância da realização de avaliações precoces com o intuito de prevenir problemas ou atrasos no desenvolvimento infantil.

A ocorrência de fatores de risco em determinada idade da criança não significa que esta irá apresentar problemas no futuro, contudo, é importante que ela receba um acompanhamento diferenciado, com consultas e avaliações de rotina. Dessa forma, é possível que alterações sejam identificadas precocemente e a família receba as devidas orientações. Também são realizadas escutas dos familiares, de modo que eles mesmos possam perceber as modificações no seu cotidiano que possam ter efeitos no desenvolvimento infantil ${ }^{2}$. Nesse sentido, os trabalhos de avaliação de risco psíquico ${ }^{3}$ sugerem a necessidade de se tomar eventuais riscos de modo indiciário e não como uma profecia negativa, pois, a partir do fato de que o desenvolvimento da criança está em curso e não consolidado, é possível pensar que tanto a plasticidade cerebral quanto interações qualificadas no ambiente podem reverter o risco. Isso é especialmente importante quando se pensa no autismo, que é considerado uma patologia congênita que afeta os movimentos intencionais e a intenção comunicativa ${ }^{4}$. Há estudos que evidenciam a possibilidade de identificação do autismo já no primeiro ano de vida ${ }^{5,6}$ e outros no segundo ano $o^{7}$ Quanto mais precoce a detecção de risco psíquico, melhores as condições para o desenvolvimento da criança: a detecção pode ser decisiva para o desenvolvimento de uma deficiência intelectual associada, por exemplo, ao risco de autismo ${ }^{8}$.

Nesse contexto, a realização de avaliações do desenvolvimento psicomotor, no campo da Fisioterapia; da linguagem e audição, no campo da Fonoaudiologia; da cognição e da constituição do psiquismo, na Psicologia, e do cotidiano infantil no campo da Terapia Ocupacional são exemplos de aspectos que interessam no estudo do desenvolvimento infantil. Especificamente em relação ao desenvolvimento motor, o método Prechtl's General Movements Assessment (GMA) é inovador e busca analisar os movimentos do bebê para a detecção de problemas 
neurológicos 9 .Já escalas como a Bayley Scales of Infant and Toddler Development (Bayley III) estão entre os melhores instrumentos de avaliação do desenvolvimento infantil, sendo mundialmente reconhecidas ${ }^{10}$.

Considerando a maior especificidade e sensibilidade do teste Bayley III, bem como os novos estudos sobre GMA, esta pesquisa avaliou bebês desde o nascimento até o segundo ano de vida. A partir da análise de filmagens realizadas durante as avaliações, foi possível observar a interação dos bebês com suas mães aos três meses e a presença ou ausência de algum movimento indicativo de problemas neurológicos, por meio do teste com o método GMA, bem como sua qualidade e relação com desfecho no desenvolvimento infantil aos 18 e 24 meses de vida dos bebês com o Bayley III.Esse acompanhamento justifica-se pela já referida maior plasticidade cerebral nessa faixa etária ${ }^{11}$ e pela possibilidade de se investigar sinais que permitissem diferenciar as demandas mais precoces para intervenção.

Portanto, o objetivo da presente pesquisa foi verificar a relação entre a avaliação do repertório motor analisada por meio do método GMA com as variáveis obstétricas (aleitamento materno; intercorrência na gestação; medicação na gestação; álcool na gestação; fumo na gestação; intercorrência ao nascer; necessidade de internação em UTI neonatal e necessidade de ventilação mecânica), a presença de risco psíquico e o desfecho no desenvolvimento da linguagem, cognitivo e motor aos 18 e 24 meses. Acredita-se que se for possível verificar algum tipo de sinal que indique risco ou atraso motor precocemente com o GMA, seria possível intervir rapidamente para evitar problemas posteriores. Dessa maneira, questiona-se se há relação entre os resultados do GMA no terceiro mês de vida com o desfecho do desenvolvimento no segundo ano de vida.

\section{MÉTODO}

\section{Local do estudo e sujeitos}

Este estudo foi realizado nas dependências do Serviço de Atendimento Fonoaudiológico de uma universidade da região central do estado do Rio Grande do Sul. A pesquisa foi realizada no período entre agosto de 2016 à março de 2017, mediante aceite do termo de consentimento livre e esclarecido pelos pais/responsáveis. As avaliações foram realizadas desde o terceiro mês de vida dos bebês e os dados anteriores ao $17^{\circ}$ mês foram coletados de forma retrospectiva, pois fizeram parte de uma pesquisa maior realizada com bebês nascidos pré-termo e a termo, provenientes do projeto "Análise comparativa do desenvolvimento de bebês prematuros e a termo, com e sem risco psíquico: da deteç̧ão à intervenção", coordenado pela autora Ana Paula Ramos de Souza. As crianças foram acompanhadas pelo projeto desde seus primeiros dias de vida, com avaliações periódicas.

\section{Tipo de estudo e amostra}

Estudo longitudinal, de caráter analítico, prospectivo e retrospectivo, com realização das avaliações do desenvolvimento infantil até os 24 meses.

A amostra foi de conveniência, a partir da demanda das avaliações do projeto mãe, no qual participou uma subamostra dessa pesquisa. Dessa forma, foram avaliados 47 bebês. Cinco destes foram excluídos por não ser possível a observação no vídeo para a análise do GMA, devido interferência de algum objeto ou mesmo do posicionamento da mãe, dificultando a visualização do bebê. Portanto, a amostra foi composta por 42 bebês, 12 prematuros e 17 nascidos a termo. A idade gestacional foi corrigida até os 24 meses nos nascidos prematuros.

\section{Procedimento de coleta de dados}

A coleta iniciou após a explicação sobre os procedimentos do estudo, que consistiam em observações e filmagens durante os dois primeiros meses de desenvolvimento. Os bebês foram captados em uma unidade básica de saúde no dia em que compareceram para a realização do teste do pezinho. Alguns bebês e seus familiares também foram convidados em setor de seguimento de prematuros extremos em um hospital universitário próximo a unidade. Em sua maioria, eram bebês nascidos a termo e prematuros tardios. Nesse momento, foi realizada uma entrevista com os familiares, com o intuito de coletar dados a respeito da história do bebê, sobretudo informações obstétricas e sobre seus cuidados iniciais. Após essa entrevista, a mãe foi orientada a retornar em outro momento para a avaliação do desenvolvimento do bebê. Essa avaliação ocorreu em cinco faixas etárias, nas quais foram realizadas as avaliações utilizadas na presente pesquisa, entre outras do projeto maior. A seguir estão descritos apenas os procedimentos desta pesquisa:

- Bebês de 3 meses e 1 dia a 4 meses e 29 dias: foram realizadas as avaliações com os sinais PREAUT (Programme de Recherche et Evaluation sur l'autisme) ${ }^{5}$; indicadores de risco ao desenvolvimento infantil $(\mathrm{IRDI})^{4}$ fase I; filmagem do bebê interagindo com 
a mãe por 15 minutos - sendo que durante seis minutos o bebê permanece em decúbito ventral e em prono. A partir dessa filmagem, foi realizada análise por meio do método GMA. Cabe destacar o caráter complementar entre sinais PREAUT e IRDI na avaliação do psiquismo ${ }^{12}$.

- Bebês de 8 meses e 1 dia a 8 meses e 29 dias: foram realizadas as avaliações com sinais PREAUT, IRDI fase II e filmagem de 15 minutos do bebê sentado com caixa de brinquedos correspondentes à faixa etária e interagindo com a mãe.

- Bebês de 11 meses e 1 dia a 12 meses e 29 dias: foram realizadas avaliações com o roteiro IRDI fase III e filmagem de 15 minutos do bebê sentado com caixa de brinquedos correspondentes à faixa etária e interagindo com a mãe.

- Bebês de 17 meses e 1 dia a 18 meses e 29 dias: foram realizadas avaliações com o roteiro IRDI fase IV; aplicação do questionário M-CHAT ${ }^{13}$; filmagem de 15 minutos do bebê sentado com caixa de brinquedos correspondentes à faixa etária e interagindo com a mãe; realização do teste Bayley $\mathrm{III}^{14}$.

- Bebês de 23 meses e 1 dia a 24 meses e 29 dias: foram realizadas avaliações com o questionário M-CHAT; filmagem de 15 minutos do bebê sentado com caixa de brinquedos correspondente à faixa etária e interagindo com a mãe; realização do teste Bayley III.

As filmagens realizadas com os bebês e as mães faziam parte do projeto maior. Para a presente pesquisa, foi utilizado um recorte apenas de momentos das filmagens em que o bebê permaneceu em decúbito ventral, quando foi possível observar seus movimentos. Os testes para detecção do risco psíquico, sinais PREAUT, roteiro IRDI e M-CHAT foram observados pelos pesquisadores durante as entrevistas e filmagens de cada faixa etária e assinalados em seus valores.

Os sinais PREAUT são específicos para captar risco para autismo quando a sua pontuação é inferior a cinco pontos e risco de outra psicopatologia quando a pontuação está entre cinco e 15 pontos. Crianças com 15 pontos são consideradas fora de risco ${ }^{4}$.

O roteiro IRDI é composto por 18 indicadores, distribuídos em quatro faixas etárias. Se o bebê tem dois ou mais indicadores ausentes, ele pode ser considerado um caso de risco aos 18 meses. Este risco pode ser ao desenvolvimento, quando não há risco de emergência de uma psicopatologia, ou risco para uma psicopatologia, como autismo ou psicose ${ }^{12}$.
O teste M-CHAT propõe-se a avaliar o risco para o transtorno do espectro autista (TEA) e é uma ferramenta cientificamente validada e projetada para a triagem de crianças de 18 e 24 meses de idade. Tratase de um questionário com 23 questões em que os pais indicam presença ou ausência de um determinado comportamento ${ }^{7,13,15}$. O teste é composto por 19 questões relativas à presença ou ausência de competências e de quatro questões relativas à presença ou ausência de comportamentos atípicos. Tratando-se de crianças típicas, a resposta esperada para as questões relativas às competências típicas é "sim"; a resposta para as questões que indicam comportamento atípico, "não". Dentre as questões do questionário, existem seis que são considerados itens críticos para um desenvolvimento autístico (questões de número 2, 7, 9, 13, 14 e 15). Dessa forma, considerase que existe um risco para o desenvolvimento autístico quando a criança falha em dois ou mais itens críticos ou em três itens que qualquer natureza ${ }^{13}$.

O método GMA aborda movimentos espontâneos do bebê que envolvem todo o corpo com uma sequência variável de movimentos de braços, pernas, pescoço e tronco, alternando em amplitude e velocidade. É realizado por meio da observação desde o nascimento até as 15 semanas pós-termo, permitindo avaliação de bebês muito prematuros logo após o nascimento, incluindo os bebês em ventilação mecânica ${ }^{16}$. $\mathrm{Na}$ presente pesquisa, foi realizado juntamente com a primeira filmagem (de 3 meses e 1 dia a 4 meses e 29 dias) por uma profissional capacitada para tal, que não tinha nenhuma informação sobre o fato de o bebê ser nascido a termo ou prematuro, ou a respeito do perfil geral da amostra. $O$ teste é baseado na observação dos movimentos espontâneos da criança sem intervenção ou estímulo externo, realizada através de filmagens da criança deitada em supino, enquanto ela está acordada, repousando na incubadora ou no leito ${ }^{17}$. Os movimentos gerais (general movements - GM) são avaliados de forma qualitativa e classificados como normais ou anormais de acordo com sua fluência, variabilidade e complexidade. Os GM normais têm início e fim graduais; eles envolvem todo o corpo em padrões complexos e variáveis de flexão, extensão e rotação que dão uma impressão de fluência e elegância. GM a partir de 10 semanas de idade pós termo são chamados de Fidgety Movements (FM) ${ }^{16}$. A organização dos FM varia com a idade, sendo que na fase inicial (de 6 a 8 semanas) surgem em momentos isolados, com aumento da frequência, e diminuem novamente após 15-18 semanas, coincidindo com o período em que os movimentos antigravitacionais e intencionais começam 
a dominar. Os FM podem ser normais (contínuos, intermitentes e esporádicos) e anormais (ausentes e esporádicos em algumas partes do corpo $)^{17}$.

O teste Bayley III foi realizado por uma fisioterapeuta formada para sua aplicação apenas entre os 18 e 24 meses dos bebês, por se tratar de teste extenso (duas sessões para a aplicação) que teve pouca adesão dos familiares. $\mathrm{Na}$ pesquisa, o foco foi o teste Bayley III para o desfecho por se entender sua importância como padrão ouro na literatura internacional ${ }^{18}$. Embora não façam parte das análises aqui apresentadas, os bebês foram acompanhados com outros testes durante as filmagens na pesquisa maior. O funcionamento da escala Bayley III foi explicado aos pais/responsáveis, e foi solicitado que estes não ajudassem e interferissem durante o teste, a menos que fosse solicitado - senão a questão poderia não ser pontuada. Inicialmente foi calculada a idade gestacional dos bebês, corrigindo quando prematuros até os 24 meses. A partir daí verificou-se o ponto de partida do teste e encontrouse a base para cada bebê (três questões corretas). O teste só era finalizado quando o bebê respondia cinco questões incorretas. $\mathrm{Na}$ avaliação com a escala Bayley III nesta pesquisa, foram utilizados três dos cinco subtestes: de desenvolvimento da linguagem, que aborda comunicação receptiva e expressiva, de desenvolvimento cognitivo e de desenvolvimento da coordenação motora fina e grossa.

\section{Variáveis em análise}

As variáveis analisadas na presente pesquisa são gestacionais e de nascimento, como o aleitamento materno, intercorrência durante a gestação, medicação durante a gestação, álcool durante a gestação, fumo durante a gestação, intercorrência ao nascer, necessidade de internação em UTI neonatal e necessidade do uso da ventilação mecânica; testes para detecção do risco psíquico, PREAUT aos 9 meses, IRDI total até os 18 meses e M-CHAT dos 18 e 24 meses; teste Bayley III aos 18 e 24 meses (cognitivo, linguagem e motor).

\section{Análise de dados}

Inicialmente, foram calculados os percentuais para as variáveis de interesse. Posteriormente, foram avaliadas as associações entre o método GMA com as variáveis obstétricas, as condições de nascimento, os testes psíquicos e o desfecho final do teste Bayley III (no segundo ano de vida), sendo utilizado o teste Exato de Fisher (para frequências pequenas) e, para o Método GMA com o aleitamento materno, o teste de associação qui-quadrado. Para a realização das análises, foi utilizado o aplicativo computacional Statistica 9.1, sendo considerado o nível de significância de $5 \%$.

\section{RESULTADOS}

A amostra foi composta por 42 bebês, $61,9 \%(n=26)$ do sexo masculino e $38,1 \%(n=16)$ do sexo feminino, sendo $40,5 \%(n=17)$ nascidos pré-termo e 59,5\% ( $n=25)$ nascidos a termo. A Tabela 1 apresenta os resultados do método GMA considerando a comparação entre nascidos a termo e pré-termo. Dos 42 bebês avaliados pelo método GMA, $29(69,0 \%)$ apresentaram normalidade na avaliação dos FM - destes $12(41,4 \%)$ foram nascidos prematuros e 17 $(58,6 \%)$ a termo. Dos $13(31,0 \%)$ bebês que apresentaram anormalidade nos FM, 5 (38,5\%) foram prematuros e 8 $(61,5 \%)$ nascidos a termo. Não houve diferença entre ser ou não prematuro na análise de GMA, já que tanto entre prematuros quanto entre nascidos a termo os percentuais foram muito próximos.

Tabela 1. Distribuição percentual do método GMA e comparação conforme idade gestacional

$\begin{array}{lrccc}\text { GMA } & \text { N (\%) } & \text { RNPT } & \text { RNT } & \text { p_valor** } \\ \text { Normal } & 29(69,0 \%) & 12(41,4 \%) & 17(58,6 \%) & 1,0000 \\ \text { Alterado/anormal } & 13(31,0 \%) & 5(38,5 \%) & 8(61,5 \%) & \end{array}$

*p valor $\leq 0,05$; **Teste Exato de Fisher; RNPT: recém-nascido pré-termo; RNT: recém-nascido a termo; GMA: Precht's General Movements Assessment; N: número; \%: porcentagem.

A Tabela 2 refere-se aos dados do método GMA e o tipo de aleitamento materno realizado, ocorrido de forma exclusiva, mista ou artificial. Conforme a análise da associação com o teste qui-quadrado, observa-se que não houve associação entre essas variáveis. A Tabela 3 apresenta o resultado entre o método GMA e as variáveis obstétricas pré e pós-natais (intercorrência na gestação, medicação na gestação, álcool na gestação, fumo na gestação, intercorrência ao nascer, UTI neonatal e ventilação mecânica). Não houve associação entre essas variáveis.

Tabela 2. Distribuição percentual do método PGM e comparação conforme o tipo de aleitamento materno

\begin{tabular}{lccccc}
\multirow{2}{*}{$\begin{array}{l}\text { Tipo de } \\
\text { aleitamento }\end{array}$} & \multicolumn{2}{c}{ GMA normal } & \multicolumn{2}{c}{$\begin{array}{c}\text { GMA alterado/ } \\
\text { anormal }\end{array}$} & P_valor** \\
\cline { 2 - 6 } & $\mathbf{N}$ & $(\%)$ & $\mathbf{n}$ & $(\%)$ & \\
Exclusivo & 13 & 46,4 & 9 & 69,3 & \\
Misto & 9 & 32,2 & 4 & 30,7 & 0,1611 \\
Artificial & 6 & 21,4 & 0 & 0 &
\end{tabular}

*p valor $\leq 0,05$; **Teste qui-quadrado; GMA: Precht's General Movements Assessment; N: número; $\%$ : porcentagem 
Tabela 3. Distribuição percentual do método GMA e comparação conforme variáveis obstétricas pré e pós-natais

\begin{tabular}{|c|c|c|c|c|c|}
\hline \multirow{2}{*}{ Variáveis } & \multicolumn{2}{|c|}{ PGM normal } & \multicolumn{2}{|c|}{ PGM alterado/ anormal } & \multirow[t]{2}{*}{ p_valor*: } \\
\hline & $\mathrm{n}$ & $(\%)$ & $n$ & $(\%)$ & \\
\hline \multicolumn{6}{|c|}{ Intercorrência na gestação ( $n=41)$} \\
\hline $\operatorname{sim}$ & 16 & $57,1 \%$ & 7 & $53,9 \%$ & 1,0000 \\
\hline Não & 12 & $42,9 \%$ & 6 & $46,1 \%$ & \\
\hline \multicolumn{6}{|c|}{ Medicação na gestação ( $n=40)$} \\
\hline Sim & 15 & $55,6 \%$ & 4 & $30,8 \%$ & 0,1861 \\
\hline Não & 12 & $44,4 \%$ & 9 & 69,23 & \\
\hline \multicolumn{6}{|c|}{ Álcool na gestação (n=38) } \\
\hline Sim & 0 & 0 & 0 & 0 & 1,0000 \\
\hline Não & 26 & $100 \%$ & 12 & $100 \%$ & \\
\hline \multicolumn{6}{|c|}{ Fumo na gestação ( $n=38)$} \\
\hline Sim & 2 & $7,7 \%$ & 0 & 0 & 1,0000 \\
\hline Não & 24 & $92,3 \%$ & 12 & $100 \%$ & \\
\hline \multicolumn{6}{|c|}{ Intercorrência ao nascer ( $n=41)$} \\
\hline Sim & 5 & $17,9 \%$ & 4 & $30,8 \%$ & 0,4288 \\
\hline Não & 23 & $82,1 \%$ & 9 & $69,2 \%$ & \\
\hline \multicolumn{6}{|c|}{ UTI neonatal $(n=41)$} \\
\hline $\operatorname{sim}$ & 5 & $17,9 \%$ & 4 & $30,8 \%$ & 0,4288 \\
\hline Não & 23 & $82,1 \%$ & 9 & $69,2 \%$ & \\
\hline \multicolumn{6}{|c|}{ Ventilação mecânica ( $n=38)$} \\
\hline Sim & 4 & $15,4 \%$ & 1 & 8,3 & 1,0000 \\
\hline Não & 22 & $84,2 \%$ & 11 & $91,7 \%$ & \\
\hline
\end{tabular}

*p valor $\leq 0,05 ;{ }^{* *}$ Teste Exato de Fisher; GM: General Movements; GMA: Prechtl's General Movements; N: número; \%: porcentagem; UTI: unidade de terapia intensiva.

$\mathrm{Na}$ Tabela 4, estão expostos os resultados entre a relação do método GMA com os testes psíquicos (PREAUT, IRDI, M-CHAT). Cada teste variou de acordo com a participação dos bebês em cada etapa do estudo. É possível observar que não houve associação estatisticamente significativa entre os resultados dos testes psíquicos e o método GMA. A Tabela 5 refere-se à análise da associação entre os resultados do método GMA e o desfecho final com o teste Bayley III aos 18 e 24 meses. Não houve associação da presença ou não de atraso no teste Bayley III com a normalidade ou anormalidade com o método GMA.

Tabela 4. Distribuição percentual da análise de associação entre o método PGM e testes psíquicos (PREAUT, IRDI, M-CHAT)

\begin{tabular}{|c|c|c|c|c|c|}
\hline \multirow{3}{*}{ Testes } & \multicolumn{4}{|c|}{ General movements } & \multirow[b]{3}{*}{ p_valor** } \\
\hline & \multicolumn{2}{|c|}{ Normal } & \multicolumn{2}{|c|}{ alterado/ anormal } & \\
\hline & $\mathrm{n}$ & $\%$ & n & $\%$ & \\
\hline \multicolumn{6}{|c|}{ PREAUT 9 meses $(n=40)$} \\
\hline Sem risco & 20 & $74,1 \%$ & 10 & $76,9 \%$ & \multirow{2}{*}{1,0000} \\
\hline Com risco & 7 & $25,9 \%$ & 3 & $23,1 \%$ & \\
\hline \multicolumn{6}{|c|}{ IRDI total $(n=42)$} \\
\hline Sem risco & 22 & $75,9 \%$ & 9 & $69,2 \%$ & \multirow{2}{*}{0,7132} \\
\hline Com risco & 7 & $24,1 \%$ & 4 & $30,8 \%$ & \\
\hline \multicolumn{6}{|c|}{ M-CHAT 18 meses $(n=35)$} \\
\hline Sem risco & 19 & $76,0 \%$ & 7 & $70,0 \%$ & \multirow{2}{*}{0,6936} \\
\hline Com risco & 6 & $24,0 \%$ & 3 & $30,0 \%$ & \\
\hline \multicolumn{6}{|c|}{ M-CHAT 24 meses $(n=37)$} \\
\hline Sem risco & 25 & $92,6 \%$ & 8 & $80,0 \%$ & \multirow{2}{*}{0,2914} \\
\hline Com risco & 2 & $7,4 \%$ & 2 & $20,0 \%$ & \\
\hline
\end{tabular}

"p valor $\leq 0,05 ;{ }^{* *}$ Teste Exato de Fisher; $\mathrm{N}$ : número; \%: porcentagem. 
Tabela 5. Distribuição percentual da análise de associação entre o método PGM e Bayley III

\begin{tabular}{|c|c|c|c|c|c|}
\hline \multirow{3}{*}{ Testes } & \multicolumn{4}{|c|}{ General movements } & \multirow[b]{3}{*}{ p_valor** } \\
\hline & \multicolumn{2}{|c|}{ Normal } & \multicolumn{2}{|c|}{ Alterado/ anormal } & \\
\hline & $\mathbf{n}$ & $\%$ & $n$ & $\%$ & \\
\hline Bayley cognitivo 18 meses ( $n=27$ ) & \multicolumn{2}{|c|}{ Média: 103,9 } & \multicolumn{2}{|c|}{ Média: 107,5 } & \multirow{3}{*}{0,5323} \\
\hline Sem atraso & 16 & $84,2 \%$ & 8 & $100 \%$ & \\
\hline Com atraso & 3 & 15,8 & 0 & 0 & \\
\hline Bayley cognitivo 24 MESES $(n=40)$ & \multicolumn{2}{|c|}{ Média: 91,9 } & \multicolumn{2}{|c|}{ Média: 96,9 } & \\
\hline Sem atraso & 16 & $59,3 \%$ & 9 & $69,2 \%$ & \multirow{2}{*}{0,7301} \\
\hline Com atraso & 11 & $40,7 \%$ & 4 & $30,8 \%$ & \\
\hline Bayley linguagem 18 MESES ( $n=27$ ) & \multicolumn{2}{|c|}{ Média: 85,1 } & \multicolumn{2}{|c|}{ Média: 91,8} & \multirow{3}{*}{0,6776} \\
\hline Sem atraso & 9 & $47,4 \%$ & 5 & $62,5 \%$ & \\
\hline Com atraso & 10 & $52,6 \%$ & 3 & $37,5 \%$ & \\
\hline Bayley linguagem 24 meses $(n=40)$ & \multicolumn{2}{|c|}{ Média: 87,7} & \multicolumn{2}{|c|}{ Média: 91,5} & \\
\hline Sem atraso & 13 & $48,2 \%$ & 8 & $61,5 \%$ & \multirow{2}{*}{0,5106} \\
\hline Com atraso & 14 & $51,8 \%$ & 5 & $38,5 \%$ & \\
\hline Bayley motor 18 meses $(n=26)$ & \multicolumn{2}{|c|}{ Média: 98,4 } & \multicolumn{2}{|c|}{ Média: 105,0} & \multirow{3}{*}{0,4738} \\
\hline Sem atraso & 18 & $94,7 \%$ & 6 & $85,7 \%$ & \\
\hline Com atraso & 1 & $5,3 \%$ & 1 & $14,3 \%$ & \\
\hline Bayley motor 24 meses $(n=40)$ & \multicolumn{2}{|c|}{ Média: 94,9} & \multicolumn{2}{|c|}{ Média: 96,6 } & \\
\hline Sem atraso & 23 & $85,2 \%$ & 10 & 76,9 & \multirow{2}{*}{0,6622} \\
\hline Com atraso & 4 & $14,8 \%$ & 3 & $23,1 \%$ & \\
\hline
\end{tabular}

*p valor $\leq 0,05$; **Teste Exato de Fisher; N: número.

\section{DISCUSSÃO}

As variáveis do aleitamento materno; intercorrência durante a gestação; medicação durante a gestação; álcool durante a gestação; fumo durante a gestação; intercorrência ao nascer; necessidade de UTI neonatal e ventilação mecânica não apresentaram resultados significativos quando analisados com a avaliação GMA. A ausência de significância parece estar relacionada ao fato de que a maior parte da amostra foi composta por prematuros tardios ( 15 bebês) e bebês nascidos a termo, basicamente sem grandes intercorrências ao nascimento, e GMA é um procedimento que possivelmente tem mostrado maior sensibilidade em casos que apresentam efeitos cumulativos de risco biológico, ambientais, e intercorrências ao nascer ${ }^{5,19-22}$.

A literatura reporta que a avaliação dos GM é um indicador confiável de avaliação da integridade do sistema nervoso jovem, sendo uma ferramenta confiável para identificar crianças com risco de déficits neuromotores ${ }^{23,24}$, comportamentais e cognitiva ${ }^{17}$. As evidências sugerem que o GMA tem maior especificidade em coortes de alto risco e para um diagnóstico de paralisia cerebral, principalmente ligados aos GM do tipo Fidgety ausentes ${ }^{25-28}$. Outros autores descreveram um padrão atípico de GM durante as primeiras 20 semanas de vida em bebês diagnosticados posteriormente com desordem do espectro do autismo $0^{29-31}$. Entretanto, vale salientar que a predição da avaliação do GM é baseada nas trajetórias de desenvolvimento desses movimentos e que uma única avaliação, de forma transversal, não revela se os GM avaliados como anormais ou esporádicos são apenas transitórios ou estarão, de fato, presentes por várias semanas, confirmando um diagnóstico. Isso sugere alguma limitação na aplicação desse método por meio da visualização de um único vídeo, como ocorreu nesta pesquisa, e sugere estudos futuros nos quais os bebês possam ser avaliados em mais de uma sessão nas primeiras semanas.

$\mathrm{O}$ número de bebês com aleitamento materno é percentualmente maior no grupo com GMA alterado $(69,3 \%)$, demonstrando que o aleitamento, ao menos inicialmente, não foi protetivo para este grupo, o que se opõe aos resultados de diversos estudos sobre os benefícios do aleitamento. No entanto, é possível, dado o baixo percentual de bebês com desenvolvimento motor alterado durante o acompanhamento destes, que o aleitamento tenha sido protetivo no curso evolutivo desses bebês com alteração inicial no GMA. O aleitamento materno, além de favorecer o vínculo entre a mãe e o bebê e trazer diversos benefícios já bem documentados à saúde infantil, mostra-se fortemente ligado à capacidade intelectual geral. Portanto, a amamentação deve ser sempre estimulada nos países em desenvolvimento, onde as crianças são expostas a vários riscos ambientais e biológicos, com grandes prevalências de doenças e até mesmo gestações desfavoráveis ou prematuras e precárias condições socioeconômicas ${ }^{32}$. 
O estudo de Spittle et al. ${ }^{33}$ teve como objetivo verificar o desenvolvimento neurológico de nascidos pré-termo por meio do método GMA no primeiro e terceiro meses de vida e de Bayley III aos dois e quatro anos. Foi evidenciado que quando os GM mostraram resultado anormal no primeiro mês de vida, os resultados do Bayley III subteste motor foram piores aos dois e quatro anos. Já a avaliação do GM no terceiro mês com resultado anormal associou-se com piores desempenhos cognitivos, de linguagem e motor aos dois e quatro anos de idade. Logo, pode-se dizer que GM anormal no terceiro mês de vida é preditivo de pior desenvolvimento neurológico. Diferentemente do estudo de Spittle et al. ${ }^{33}$, os resultados desta pesquisa não mostraram resultado significativo entre a associação entre o teste Bayley III com o método GMA. Esse fato também pode ser explicado com base no perfil da amostra que participou da coleta do GM, visto que esta difere bastante dos estudos já publicados.

Fidgety Movements (movimentos irregulares) anormais, ausentes ou esporádicos indicam predisposição maior de disfunções neurológicas posteriores quando comparados aos normais, principalmente quando acompanhados de outros movimentos suaves e fluentes. $\mathrm{O}$ reconhecimento precoce desses sinais neurológicos facilita uma intervenção precoce, principalmente quando se trata de neonatos que estão mais expostos aos fatores de risco ${ }^{17}$. Nesta pesquisa, não verificamos o mesmo tipo de alteração desses estudos, em função da falta de relação entre as variáveis na amostra estudada entre os desfechos em cognição, linguagem e motricidade no teste Bayley III com a avaliação realizada no GMA.

Cabe destacar que a facilidade de aplicação do método GMA sobre filmagens, desde que haja um profissional fisioterapeuta habilitado para observar os vídeos, permite indicar que se trata de tecnologia de baixo custo e que pode integrar o sistema de telessaúde, o que é desejável em um país com a extensão territorial do Brasil e com pequeno número de fisioterapeutas na atenção primária. A inexistência de uma carreira em saúde cria dificuldades importantes para que o conhecimento científico das profissões que estudam desenvolvimento infantil chegue na puericultura, que ainda é muito limitada ao crescimento físico, nutrição e detecção de doenças na realidade brasileira. No entanto, a presença de fisioterapeutas nos núcleos de assistência à estratégia de saúde da família, poderia se valer do método GMA como forma de seleção dos bebês que demandem uma avaliação mais específica do profissional de fisioterapia.

\section{CONCLUSÃO}

Com base nos resultados desta pesquisa, verificou-se que não houve relação entre o método GMA e as variáveis analisadas. Acredita-se que, pelo fato de a amostra ter sido composta, em sua maioria, por bebês nascidos a termo ou prematuros tardios sem intercorrências e de a avaliação ter sido de forma transversal, em um único momento, não foi possível analisar se os movimentos avaliados como anormais foram ou não transitórios. Esses resultados contribuem para reforçar a importância da realização de avaliações longitudinais dos bebês que de fato são considerados de risco, seja pelo nascimento prematuro ou por algum outro tipo de intercorrência.

A limitação na amostra deste estudo ocorreu em razão da perda de sujeitos relacionada à impossibilidade de comparecer a duas avaliações do Bayley III, pois uma pesquisa com amostra maior seria mais consistente para ocorrer a generalização dos resultados. No entanto, esta pesquisa enfatiza a importância para o uso do método GMA nos casos de prematuridade extrema e em bebês que passaram por intercorrências antes, durante e após o nascimento. As limitações organizacionais da puericultura na realidade brasileira demandam que as equipes façam escolhas dos métodos mais eficazes para cada população. A vantagem do GMA é a possibilidade da sua utilização sobre filmagens, sem nenhum estresse ao bebê, salvaguardadas a idade do bebê e a qualidade das filmagens para sua visualização - algo que, com o acesso a tecnologias, como a dos celulares, é facilitado atualmente.

\section{REFERÊNCIAS}

1. Weiss LG, Oakland T, Aylward GP. Bayley-III: uso clínico e interpretação. São Paulo: Pearson Clinical Brasil 2017.

2. Mello RR, Meio MDBB. Follow up de recém-nascidos de risco. In: Moreira MEL, Braga NA, Morsch S, organizadores. Quando a vida começa diferente: o bebê e sua família na UTI neonatal. Rio de Janeiro: Fiocruz; 2003. p. 179-84.

3. Kupfer MC, Jerusalinsky NA, Bernardino LMF, Wanderley D, Rocha PSB, Molina SE, et al. Predictive value of clinical risk indicators in child development: final results of a study based on psychoanalytic theory. Rev Latinoam Psicopat Fund. 2010;13(2):31-52. doi: 10.1590/S1415-47142010000100003

4. Trevarthen C, Delafield-Butt JT. Autism as a developmental disorder in intentional movement and affective engagement. Front Integr Neurosci. 2013;7:1-16. doi: 10.3389/fnint.2013.00049

5. Olliac B, Crespin G, Laznik MC, El Ganouni OCl, Sarradet $\mathrm{JL}$, Bauby $\mathrm{C}$, et al. Infant and dyadic assessment in early community-based screening for autism spectrum disorder 
with the PREAUT grid. PLOS ONE. 2017;12(12):e0188831. doi: 10.1371/journal.pone.0188831

6. Ouss L, Saint-Georges C, Roel L, Bodeau N, Laznik MC, Crespin GC, et al. Infant's engagement and emotion as predictors of autism or intelectual disability in West Syndrome. Eur Child Adolesc Psychiatry. 2014;23(3):143-9. doi: 10.1007/ s00787-013-0430-x

7. Robins DL, Fein D, Barton ML, Green JA. The modified checklist for autism in toddlers: an initial study investigating the early detection of autism and pervasive developmental disorders. J Autism Dev Disord. 2001;31(2):131-44. doi: 10.1023/a:1010738829569

8. Trevarthen C. Sharing joyful friendship and imagination for meaning with infants, and their application in early intervention. In: Acquarone S, editora. Surviving the early years: the importance of early intervention with babies at risk. London: Karnak Books; 2016. p. 19-26.

9. Einspieler C, Prayer D, Prechtl HFR. Fetal behaviour: a neurodevelopmental approach. London: Mac Keith; 2012.

10. Jackson BJ, Needelman H, Roberts H, Willet S, McMorris C. Bayley scales of infant development screening test-gross motor subtest: efficacy in determining need for services. Pediatr phys ther. 2012;24(1):58-62. doi: 10.1097/PEP.0b013e31823d8ba0

11. Spittle AJ, Olsen J, Kwong A, Doyle LW, Marschik PB, Einspieler $C$, et al. The baby moves prospective cohort study protocol: using a smartphone application with the General Movements Assessment to predict neurodevelopmental outcomes at age 2 years for extremely preterm or extremely low birthweight infants. BMJ Open. 2016;6(10):e013446. doi: 10.1136/ bmjopen-2016-013446

12. Hoogstraten AMRJ, Souza APR, Moraes AB. A complementaridade entre sinais PREAUT e IRDI na análise de risco psíquico aos nove meses e sua relação com idade gestacional. CoDAS. 2018;30(5):e20170096. doi: 10.1590/2317-1782/20182017096

13. Muratori F. O diagnóstico precoce do autismo: guia prático para pediatras. Salvador: NIIP; 2014.

14. Bayley N. Bayley scales of infant and toddler development. 3rd ed. London: Pearson; 2005.

15. Losápio MF, Pondé MP. Tradução para o português da escala M-CHAT para rastreamento precoce de autismo. Rev Psiquiatr. 2008;30(3):221-9. doi: 10.1590/S0101-81082008000400011

16. Einspieler C, Prechtl HFR. Prechtl's assessment of general movements: a diagnostic tool for the functional assessment of the young nervous system. Ment Retard Dev Disabil Res Rev. 2005;11(1):61-7. doi: 10.1002/mrdd.20051

17. Einspieler C, Peharz R, Marschik PB. Fidgety movement: tiny in appearance, but huge in impact. J Pediatr. 2016;92(3 Suppl 1):564-70. doi: 10.1016/j.jped.2015.12.003

18. Bradley-Johnson S, Johnson M. Infant and toodler assessment. In: Bracken BA, Nagle RJ, editores. Psycho educational assessment of preschool children. 4th ed. Mahwah: Lawrence Erlbaum Associates; 2007. p. 325-57.

19. Zajonz R, Müller AB, Valentini NC. The influence of environmental factors in motor and social performance of children from the suburb of Porto Alegre. J Phys Educ. 2008;19(2):159-71. doi: 10.4025/reveducfis.v19i2.3220
20. Saccani R, Valentini NC, Pereira KR, Müller AB, Gabbard C. Associations of biological factors and affordances in the home with infant motor development. Pediatr Int. 2013;55(2):197-203. doi: 10.1111/ped.12042

21. Pereira KRG, Saccani R, Valentini NC. Cognição e ambiente são preditores do desenvolvimento motor de bebês ao longo do tempo. Fisioter Pesqui. 2016;23(1):59-67. doi: 10.1590/1809-2950/14685223012016

22. Borba LS, Pereira KRG, Valentini NC. Motor and cognitive development predictors of infants of Adolescents and adults mothers. J Phys Educ. 2017;28(1):e281. doi: 10.4025/jphyseduc. v28i1.2811

23. Crowle C, Walker K, Galea C, Novak I, Badawi N. General movement trajectories and neurodevelopment at 3 months of age following neonatal surgery. Early Hum Dev. 2017;111:42-8. doi: 10.1016/j.earlhumdev.2017.05.010

24. Einspieler C, Marschik PB, Bos AF, Ferrari F. Early markers for cerebral palsy: insights from the assessment of general movements. Future Neurol. 2012;7(6):709-17. doi: 10.2217/fnl.12.60

25. Prechtl HF, Einspieler C, Cioni G, Bos AF, Ferrari F, Sontheimer D. An early marker for neurological deficits after perinatal brain lesions. Lancet. 1997;349(9062):1361-3. doi: 10;349(9062):1361-3

26. Bosanquet M, Copeland L, Ware R, Boyd R. A systematic review of tests to predict cerebral palsy in young children. Dev Med Child Neurol. 2013;55(5):418-26. doi: 10.1111/dmcn.12140

27. Grunewaldt KH, Fjørtoft T, Bjuland KJ, Brubakk AM, Eikenes L, Håberg AK, et al. Follow-up at age 10 years in ELBW children functional outcome, brain morphology and results from motor assessments in infancy. Early Hum Dev. 2014;90(10):571-8. doi: 10.1016/j.earlhumdev.2014.07.005

28. Einspieler C, Yang H, Bartl-Pokorny KD, Chi X, Zang FF, Marschik PB, et al. Are sporadic fidgety movements as clinically relevant as is their absence. Early Hum Dev. 2015;91(4):247-52. doi: 10.1016/j.earlhumdev.2015.02.003

29. Phagava H, Muratori F, Einspieler C, Maestro S, Apicella F, Guzzetta A, et al. General movements in infants with autism spectrum disorders. Georgian Med News. 2008 [cited 2021 Mar 10];156:100-5. Disponível em: https://www.ncbi.nlm.nih. gov/pubmed/18403821

30. Palchik AB, Einspieler C, Evstafeyeva IV, Talisa VB, Marschik PB. Intra-uterine exposure to maternal opiate abuse and HIV: the impact on the developing nervous system. Early Hum Dev. 2013;89(4):229-35. doi: 10.1016/j.earlhumdev.2013.02.004

31. Zappella M, Einspieler C, Bartl-Pokorny KD, Krieber M, Coleman M, Bölte S, et al. What do home videos tell us about early motor and socio-communicative behaviours in children with autistic features during the second year of life: an exploratory study. Early Hum Dev. 2015;91(10):569-75. doi: 10.1016/j. earlhumdev.2015.07.006

32. Fonseca AL, Albernaz EP, Kaufmann CC, Neves IH, Figueiredo VLM. Impact of breastfeeding on the intelligence quotient of eight-year-old children. J Pediatr. 2013;89(4):346-53. doi: 10.1016/j.jped.2012.12.010

33. Spittle AJ, Spencer-Smith MM, Cheong JL, Eeles AL, Lee $\mathrm{KJ}$, Anderson PJ, et al. General movements in very preterm children and neurodevelopment at 2 and 4 years. Pediatrics. 2013;132(2):452-8. doi: 10.1542/peds.2013-0177 